\title{
Análise do polimorfismo genético do FOXP3 (forkhead box P3[Homo sapiens]) em indivíduos saudáveis da região Norte do Paraná Analysis of genetic polymorphism of FOXP3 (forkhead Box P3 [Homo sapiens]) in healthy individuals of Northern Paraná
}

\author{
Bruna Karina Banin Hirata ${ }^{1}$; Julie Massayo Maeda Oda²; Sueli Donizete Borelli ${ }^{3}$, \\ Maria Angelica Ehara Watanabe ${ }^{4}$
}

\begin{abstract}
Resumo
As células T regulatórias desempenham um papel central na indução e manutenção da tolerância imunológica. Expressam um fator de transcrição denominado forkhead transcription factor 3 (FOXP3), no qual polimorfismos genéticos podem levar a falta de funcionalidade destas células. Dessa forma, o objetivo do presente trabalho foi avaliar a freqüência dos polimorfismos -924 (A/G, rs2232365) e -3279 (C/A, rs3761548) do gene FOXP3 na população da região Norte do Paraná. Foi utilizada a técnica de reação em cadeia da polimerase alelo específico de 115 doadoras saudáveis e a análise foi realizada por eletroforese em gel de poliacrilamida. A população da região Norte do Paraná apresentou $8,70 \%$ do genótipo AA, 49,56\% AC e 41,74\% CC, para o polimorfismo -3279, enquanto para o polimorfismo -924, a ocorrência foi de $34,78 \%$ para o genótipo AA, $28,70 \%$ AG e 36,52\% GG. A ocorrência do alelo A para o polimorfismo -3279 foi de $33,48 \%$ e do alelo C foi de $66,52 \%$. Enquanto para o polimorfismo -924 , a ocorrência do alelo A foi de $49,13 \%$ e do alelo $\mathrm{G}$ foi de $50,87 \%$. Espera-se que, os resultados deste trabalho contribuam no desenvolvimento de estudos de associação entre os polimorfismos genéticos do gene $F O X P 3$, uma vez que o estabelecimento da genotipagem da população controle é fundamental nas análises.
\end{abstract}

Palavras-chave: FOXP3. Células T regulatórias. Polimorfismo genético.

\begin{abstract}
Regulatory T cells (Tregs) play a central role in induction and maintenance of immunological tolerance. These cells express a transcription factor called forkhead transcription factor 3 (FOXP3) in which genetic polymorphisms can lead to lack of function of these cells. Thus, the objective of this study was to evaluate the occurrence of polymorphisms -924 (A/G, rs2232365) and -3279 (C/A, rs3761548) of the FOXP3 gene in the population of Northern of Paraná. Polymorphisms were amplified by polymerase chain reaction allele-specific in 115 women healthy donors and the analysis was performed by polyacrylamide gel electrophoresis. The population of Northern of Paraná showed $8.70 \%$ of genotype $\mathrm{AA}, 49.56 \% \mathrm{AC}$ and $41.74 \% \mathrm{CC}$, for the -3279 polymorphism, while for the -924 polymorphism, the occurrence of subjects was $34.78 \%$ for genotype AA, $28.70 \%$ AG and $36.52 \%$ GG. The presence of allele A -3279 polymorphism was $33.48 \%$ and the allele C was $66.52 \%$. For the polymorphism -924 , the occurence of allele A was $49.13 \%$ and to allele $G$ was $50.87 \%$. It is hoped that the results of this study
\end{abstract}

${ }^{1}$ Laboratório de Genética Molecular e Imunologia, Departamento de Ciências Patológicas, Universidade Estadual de Londrina, Brasil. E-mail: bruna-kbh@hotmail.com.

${ }^{2}$ Laboratório de Genética Molecular e Imunologia, Departamento de Ciências Patológicas, Universidade Estadual de Londrina, Brasil. Contato principal para correspondência. E-mail: julie_massayo@hotmail.com.

${ }^{3}$ Docente lotado no Departamento de Análises Clínicas, Universidade Estadual de Maringá (UEM), Brasil. E-mail: sdborelli@uem. br.

${ }^{4}$ Docente lotado no Departamento de Ciências Patológicas, Laboratório de Genética Molecular e Imunologia, Centro de Ciências Biológicas, Universidade Estadual de Londrina (UEL), Brasil. E-mail: maewatuel@gmail.com 
contribute in the development of studies of association between genetic polymorphisms of the FOXP3 gene, since the establishment of genotyping of the population control is crucial in the analysis.

Keywords: FOXP3. Regulatory T cell. Genetic polymorphism.

\section{Introdução}

As células $\mathrm{T}$ regulatórias (Tregs) são uma população de células $T$ que atuam na tolerância imunológica e são moduladoras essenciais na resposta imune a patógenos, alérgenos, células cancerígenas e antígenos próprios, uma vez que estas induzem a supressão das células $\mathrm{T}$ efetoras, bloqueando sua ativação e função. Deficiência no desenvolvimento ou na função das Tregs são as principais causas de doenças autoimunes e inflamatórias em humanos e animais (MELO e CARVALHO, 2009; SAKAGUCHI et al., 2008).

Têm sido descritos vários mecanismos de supressão que as Tregs podem exercer sobre células $\mathrm{T}$ efetoras, tais como: contato célula-célula de maneira independente de citocinas (BACCHETTA et al. 2005); supressão através de citocinas inibitórias como IL-10 e fator de crescimento de transformação (TGF- $\beta$ ) (JONULEIT e SCHIMITT, 2003; SAKAGUCHI, 2006); destruição de adenosina ou interferência por IL-2 ou co-estimulação da via de expressão do CTLA-4 (SHEVACH, 2009). Estudos realizados por Davidson e Shevach (2011) indicaram que as células Tregs podem também alterar o potencial de migração das células T. Segundo estes autores, as Tregs limitariam o número de células $\mathrm{T}$ potencialmente auto-reativas fixando-as nos linfonodos, e impedindo desta forma que as mesmas migrem para os tecidos onde podem causar danos.

As células Tregs $\mathrm{CD}^{+} \mathrm{CD} 25^{+}$expressam o gene regulador $F O X P 3$, o qual contém 11 éxons e está localizado no braço curto $(\mathrm{p})$ do cromossomo $\mathrm{X}$, (Xp11.23). Este gene codifica um fator de transcrição denominado forkhead transcription factor 3 - FOXP3 (COFFER e BURGERING,
2004; FONTENOT et al., 2003; HORI; NOMURA; SAKAGUCH, 2003; KHATTRI et al., 2003).

$\mathrm{O}$ fator de transcrição FOXP3 tem um papel na regulação do desenvolvimento e na função das células $\mathrm{T}$ regulatórias, e a perda da expressão deste fator diminui a capacidade supressora das Tregs. A expressão de FOXP3 é regulada por mecanismos complexos, podendo ser induzida em células $\mathrm{T}$ efetoras que não possuem atividade supressora, por meio da ativação simultânea de múltiplos fatores de transcrição. Essa ativação ocorre através de receptores de células $\mathrm{T}$ (TCR) e receptores de citocinas, incluindo o receptor do TGF- $\beta$ (ALLAN et al., 2007; CHEN e OPPENHEIM, 2011; HOFFMANN et al., 2009; TONE e GREENE, 2011). Algumas Tregs podem perder a expressão do FOXP3 e converterse para células Th em resposta a determinados estímulos extrínsecos, mas o mecanismo pelo qual esta conversão ocorre ainda não é bem estabelecida, portanto a plasticidade destas células ainda é controversa (HORI, 2010).

Polimorfismos no gene $F O X P 3$ podem levar a falta de funcionalidade das Tregs $\mathrm{CD} 4{ }^{+} \mathrm{CD} 25^{+}$, podendo causar doenças autoimunes sistêmicas (BAN et al., 2007; WILDIN; SMYK-PEARSON; FILIPOVICH, 2002). Segundo Rudensky (2011) a escassez de Tregs, devido a mutações no gene $F O X P 3$ é responsável por lesões inflamatórias em camundongos e humanos, altamente agressivas. Em humanos, pelo menos 20 mutações foram identificadas no gene FOXP3 e têm sido associadas à doenças linfoproliferativas e à uma desordem autoimune denominada IPEX (immune dysregulation, polyendocrinopathy, enteropathy, X-linked syndrome) (VAN DER VLIET E NIEUWENHUIS, 2007). 
Têm sido desenvolvidos vários estudos relacionados a polimorfismos do gene FOXP3 em determinadas doenças, tais como: diabetes tipo 1 (BASSUNY et al., 2003; BJORNVOLD et al., 2006; NAKANISHI; SHIMA, 2007; ZAVATTARI et al., 2004); atopias (BOTTEMA et al., 2010); artrite idiopática juvenil (EASTELL et al., 2007); rinite alérgica (FODOR et al., 2011); doença autoimune da tireóide (BAN et al., 2007); psoríase (GAO et al., 2010); doença de Crohn e cirrose biliar primária (PARK et al. 2005); câncer de mama (RASKIN; RENNERT; GRUBER, 2009); doença de Graves e doença autoimune de Addison (OWEN et al., 2006), dentre outras.

Polimorfismos de base única (SNP - Single Nucleotide Polymorphism) têm sido descritos em várias regiões no gene $F O X P 3$, tais como região promotora, região intrônica ou não codificante e região exônica ou codificante. Os polimorfismos avaliados no presente trabalho, -924 (A/G, rs2232365) e -3279 (C/A, rs3761548), estão localizados na região promotora do gene $F O X P 3$ (GAO et al., 2010).

Os polimorfismos do gene FOXP3 (-924 e -3279) estão relacionados à rinite alérgica em resposta a ácaros da poeira doméstica e a pólen (FODOR et al., 2011; ZHANG et al., 2009), ao desenvolvimento e a intratabilidade da doença de Graves (INOUE et al., 2010) e ao baixo nível de dsDNA em mulheres com lúpus sistêmico eritematoso (LIN et al., 2011). Outros trabalhos também revelam que o polimorfismo -3279 não está relacionado com endometriose, doença de Crohn e risco de desenvolvimento de câncer de mama (ANDRÉ et al., 2011; PARK et al., 2005; RASKIN; RENNERT; GRUBER, 2009).

Embora haja vários trabalhos relacionados à associação desses polimorfismos com doenças, não há uniformidade na distribuição dos genótipos dentro da população saudável usada como grupo controle.

Diante do exposto, o presente trabalho tem como objetivo avaliar a ocorrência dos polimorfismos -924 (A/G, rs2232365) e -3279 (C/A, rs3761548) do gene $F O X P 3$ de células T regulatórias na população da região Norte do Paraná.

\section{Material e Métodos}

Aspectos éticos

Este projeto foi aprovado pelo Comitê de Ética em Pesquisa em Seres Humanos - Universidade Estadual de Londrina, CAAE - 0179.0.268.00009, o qual está de acordo com a resolução 196/96 - CNS. Todos os participantes do projeto assinaram o Termo de Consentimento Livre e Esclarecido.

\section{Doadores saudáveis}

Participaram deste projeto 115 doadoras saudáveis da Região Norte do Paraná. Todos os indivíduos apresentaram sorologia negativa do HIV, hepatites B e C, HTLV (Human T-Tymphotropic Virus), sífilis e Chagas.

\section{Extração de DNA}

O DNA genômico dos doadores saudáveis foi extraído a partir de leucócitos do sangue periférico pelo kit Qiamp spin Column (Quigen Inc. Chatswcth, CA), de acordo com instruções do fabricante. A concentração do DNA foi ajustada para $100 \mathrm{ng} / \mu \mathrm{L}$ com água milli-Q (BORELLI et al, 2004). As amostras de DNA foram quantificadas por espectrofotometria no aparelho UV-1650PC (Shimadzu, Kyoto, Japan) nos comprimentos de onda de $260 \mathrm{~nm}$ e $280 \mathrm{~nm}$. A concentração do DNA em ng/ $\mu \mathrm{L}$ foi calculada utilizando-se $\mathrm{ng} / \mu \mathrm{L}$ $=\operatorname{Abs} 260$ x FD x 50, considerando-se que uma unidade de absorbância a $260 \mathrm{~nm}$ equivale a 50 $\mu \mathrm{g} \mathrm{DNA} / \mathrm{mL}$, e FD o fator de diluição. O grau de 
pureza em relação à contaminação por proteínas foi avaliado pela razão entre as absorbâncias nos comprimentos de $260 \mathrm{~nm}$ e $280 \mathrm{~nm}$ (260/280). Foram utilizadas nos experimentos amostras de DNA que apresentaram relações de absorbâncias maiores ou iguais a $1,7(260 / 280 \geq 1,7)$ (WHITE; DE LUCA, 1977).

Análise do polimorfismo FOXP3 (-924, -3279)

Os iniciadores utilizados para a amplificação da região promotora do gene FOXP3 foram sintetizados de acordo com a sequência do GenBank NT_079573.4 para os polimorfismos -924 (A/G, rs2232365) e -3279 (C/A, rs3761548).

Aproximadamente 100ng de DNA foram utilizados para amplificar, pela reação em cadeia da polimerase (PCR). As amostras foram amplificadas utilizando 1,25U de Taq polimerase (InvitrogenTM, Carlsbad, California, USA). As reações de amplificação para os polimorfismos -3279 (C/A) e -924 (A/G) foram realizadas em termociclador (PCR-Sprint Hybaid - Guelph, Ontario, Canada). As condições da reação de amplificação foram realizadas de acordo com o seguinte protocolo: 1 min a $96^{\circ} \mathrm{C}$, seguida de 5 ciclos de $96^{\circ} \mathrm{C}$ por 20 seg e $70^{\circ} \mathrm{C}$ por 45 seg. Subsequentemente, houve 21 ciclos de $96^{\circ} \mathrm{C}$ por $25 \mathrm{seg}, 65^{\circ} \mathrm{C}$ por $50 \mathrm{seg}$ e $72^{\circ} \mathrm{C}$ por 30 seg, seguidos de outros 21 ciclos a $96^{\circ} \mathrm{C}$ por $30 \mathrm{seg}$, $55^{\circ} \mathrm{C}$ por $60 \mathrm{seg}$ e $72^{\circ} \mathrm{C}$ por $90 \mathrm{seg}$. Após o último ciclo, ocorreu a extensão final de 2 minutos a $72^{\circ} \mathrm{C}$ (GAO et al., 2010). Os fragmentos amplificados para FOXP3 -924 de 442pb (A) e 427pb (G), assim como os para FOXP3 -3279 de 334pb (A) e 333pb (C) foram analisados por eletroforese em gel de poliacrilamida $(10 \%)$ e corados com Nitrato de Prata $\left(\mathrm{AgNO}_{3}\right)$.

\section{Resultados e Discussão}

Os polimorfismos -3279 e -924 do gene FOXP3 apresentaram três genótipos distintos. Para o polimorfismo -924, 34,78\% dos indivíduos eram genótipo AA, 28,70\% AG e 36,52\% GG. Enquanto para o polimorfismo $-3279,8,70 \%$ dos indivíduos apresentaram genótipo AA, 49,56\% eram AC e 41,74\% eram CC, como pode ser visto na Figura 1. A frequência do alelo A para o polimorfismo -3279 foi de $33,48 \%$ e do alelo $\mathrm{C}$ foi de $66,52 \%$. Enquanto para o polimorfismo $-924,49,13 \%$ dos indivíduos possuíam o alelo A e 50,87\% possuíam o alelo $\mathrm{G}$.

Figura 1 - Distribuição genotípica dos polimorfismos genéticos -924 (A/G, rs2232365) e -3279 (C/A, rs3761548) dentro da amostra total de indivíduos estudados

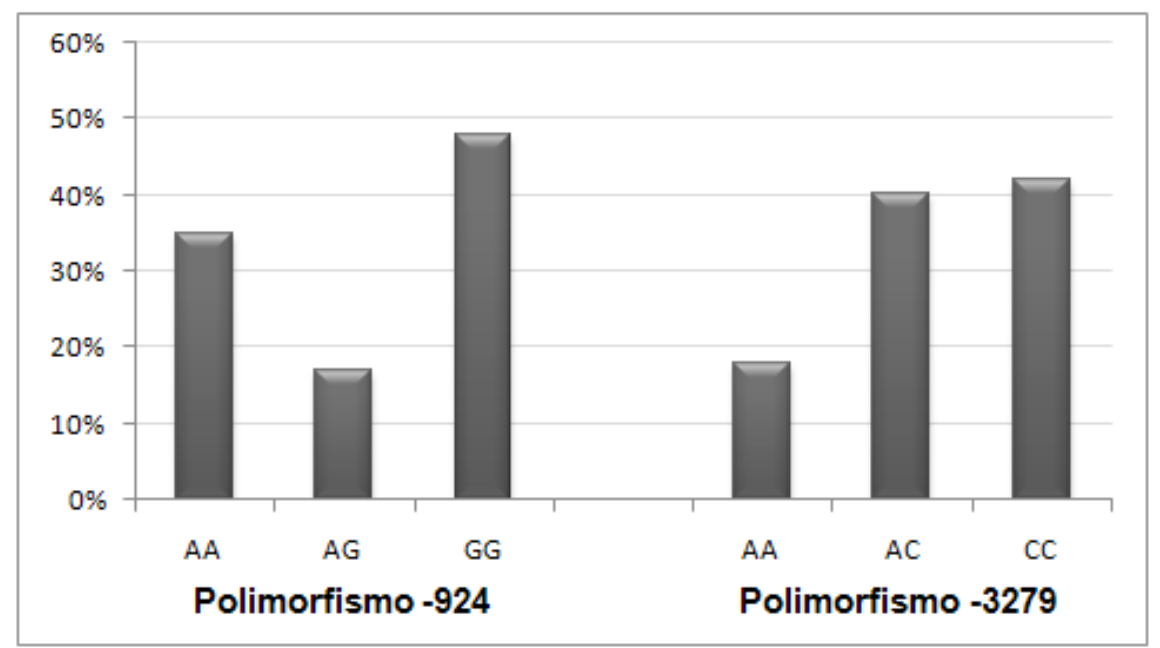

Fonte: Autores. 
Os resultados deste trabalho foram comparados aos de estudos prévios, um realizado também com indivíduos da população brasileira e os demais realizados com outras populações tais como o da China, Estados Unidos da América, Inglaterra e
Taiwan. As freqüências alélicas e genotípicas para a região Norte do Paraná apresentaram menor taxa de indivíduos em heterozigose, sendo que a frequência alélica foi similar ao trabalho de $\mathrm{Wu}$ et al. (2012).

Tabela 1 - Comparação do polimorfismo -924 do gene FOXP3 entre o presente estudo e outros trabalhos.

\begin{tabular}{lcccccc}
\hline \multicolumn{7}{c}{ Polimorfismo -924 } \\
Lutores & \multirow{2}{*}{ Local } & \multicolumn{2}{c}{ Frequência genotípica } & \multicolumn{2}{c}{ Frequência alélica } \\
& & AA & AG & GG & Alelo A & Alelo G \\
\hline Hirata et al. & Brasil & $35 \%$ & $17 \%$ & $48 \%$ & $43,5 \%$ & $56,5 \%$ \\
Owen et al. (2006) & Inglaterra & $*$ & $*$ & $*$ & $37,5 \%$ & $62,5 \%$ \\
Gao et al. (2010) & China & $37,7 \%$ & $44,6 \%$ & $17,7 \%$ & $60 \%$ & $40 \%$ \\
Wu et al. (2012) & China & $15,18 \%$ & $50 \%$ & $34,82 \%$ & $40,18 \%$ & $59,82 \%$ \\
Park (2005) & USA & $22 \%$ & $53 \%$ & $25 \%$ & $*$ & $*$ \\
\hline
\end{tabular}

* valores não citados pelos autores.

Fonte: Autores.

Tabela 2 - Comparação do polimorfismo -3279 do gene FOXP3 entre o presente estudo e outros trabalhos.

\begin{tabular}{llccccc}
\hline \multicolumn{7}{c}{ Polimorfismo -3279 } \\
\hline \multirow{2}{*}{ Autores } & \multirow{2}{*}{ Local } & \multicolumn{2}{c}{ Frequência genotípica } & \multicolumn{2}{c}{ Frequência alélica } \\
& & AA & AC & CC & Alelo A & Alelo C \\
\hline \multirow{2}{*}{ Hirata et al. } & Brasil & $18 \%$ & $40 \%$ & $42 \%$ & $38 \%$ & $62 \%$ \\
André et al. (2011) & Brasil & $11,1 \%$ & $36,8 \%$ & $52 \%$ & $29,5 \%$ & $70,5 \%$ \\
Wu et al. (2012) & China & $37,5 \%$ & $40,18 \%$ & $22,32 \%$ & $57,6 \%$ & $40,4 \%$ \\
\hline
\end{tabular}

Fonte: Autores.

O trabalho realizado por Wu et al. (2012) mostrou associação significativa dos SNPs -924 e -3279 com abortamento espontâneo recorrente na população chinesa, ressaltando a importância da função do FOXP3 em uma gravidez bem sucedida. Estes autores avaliaram um grupo controle composto por
112 mulheres. A genotipagem dos polimorfismos -924 (A/G, rs2232365A/G) e rs5902434del/ATT foi realizada por PCR com primers sequênciaespecíficas, enquanto que a genotipagem dos polimorfismos -3279 (C/A, rs3761548A/C) e rs 2294021T/C foi realizada através de PCR-RFLP. 
Park et al. (2005) verificaram a existência de SNPs no gene FOXP3 humano e detectou 10 candidatos a SNPs na região promotora e intrônica, utilizando técnica de sequenciamento direto do PCR, segundo Bassuny et al. (2003).

As ocorrências do alelo A e G do polimorfismo -924 para os indivíduos da região Norte do Paraná são compatíveis com os resultados de Owen et al. (2006) (alelo A: 37,5\%; alelo G: 62,5\%), porém divergem dos resultados obtidos por Gao et al. (2010), segundo os quais a ocorrência do alelo A foi de $60 \%$ e do alelo $\mathrm{G}$ de $40 \%$. Quanto à distribuição dos genótipos do polimorfismo -924 houve divergências com relação a estudos prévios, como o realizado por $\mathrm{Wu}$ et al. (2012) (AA: 15,18\%; AG: 50\%; GG: 34,82\%) e por Park et al. (2005) (AA: 22\%; AG: 53\%; GG: 25\%). Embora as freqüências dos genótipos AG (44,6\%) e GG (17,7\%) obtidos por Gao et al. (2010) tenham mostrado relação inversa à obtida neste trabalho, a freqüência do genótipo AA $(37,7 \%)$ foi próxima a encontrada neste estudo $(34,78 \%)$.

As frequências alélicas e genotípicas do polimorfismo -3279 para os indivíduos da região Norte do Paraná, assemelharam-se as obtidas pelo trabalho de André et al. (2011), o qual avaliou uma população controle da região de Santo André (São Paulo, Brasil). Os autores avaliaram os polimorfismos -3279 (C/A, rs3761548) e -3283 $(\mathrm{C} / \mathrm{T}$, rs3761549) localizados na região promotora do gene FOXP3, o -20 (G/A, rs2232368) na região do intron 1 e rs2232366 (Gp87T) no intron 5, através de PCR-TaqMan. Esses autores obtiveram frequência de $29,5 \%$ para o alelo A e 70,5\% para o alelo C, uma diferença aproximada de 4\%, para ambos os alelos, das frequências obtidas no presente trabalho. Assim como as frequências alélicas, as frequências genotípicas dos indivíduos da região Norte do Paraná também se aproximaram das obtidas por André et al. (2011) (AA: 11,1\%; AC: 36,8\%; CC: 52,0\%), trabalhos realizados com a população brasileira.
Entretanto houve discrepância quando comparadas com outros estudos, como o de Wu et al. (2012), cujo trabalho foi realizado com a população chinesa, onde a freqüência do alelo A foi de $57,6 \%$ e do alelo $\mathrm{C}$ de $40,4 \%$.

\section{Conclusão}

Este estudo demonstrou que a população da região Norte do Paraná apresentou 8,70\% do genótipo AA, 49,56\% eram AC e 41,74\% eram $\mathrm{CC}$, para o polimorfismo -3279 , enquanto para o polimorfismo $-924,34,78 \%$ dos indivíduos eram genótipo AA, 28,70\% AG e 36,52\% GG. Em relação aos alelos para o polimorfismo -3279, 33,48\% dos indivíduos apresentaram o alelo A e 66,52\% possuíam o alelo C. Enquanto para o polimorfismo -924, 49,13\% dos indivíduos possuíam o alelo A e $50,87 \%$ apresentaram o alelo $\mathrm{G}$.

Até o momento, são poucos trabalhos relacionados a polimorfismos genéticos do FOXP3 cujos resultados ainda são discrepantes. Embora muitos autores tenham utilizado diferentes técnicas de PCR, ainda será necessária uma padronização mais adequada, talvez com a utilização de uma temperatura de anelamento superior à utilizada para que ocorra maior especificidade. Espera-se que este trabalho contribua para o desenvolvimento de outros estudos a fim de associar os polimorfismos genéticos do gene FOXP3 a doenças, uma vez que o estabelecimento da genotipagem da população controle é fundamental nas análises.

\section{Referências}

ALLAN, S. E.; CROME, S. Q.; CRELLIN, N. K.; PASSERINI, L.; STEINER, T. S.; BACCHETTA, R.; RONCAROLO, M. G.; LEVINGS, M. K. Activationinduced FOXP3 in human $\mathrm{T}$ effector cells does 
not suppress proliferation or cytokine production. International Immunology, Oxford, v. 19, n. 4, p. 345354, 2007.

ANDRÉ, G. M.; BARBOSA, C. P.; TELES, J. S.; VILARINO, F. L.; CHRISTOFOLINI, D. M.; BIANCO, B. Analysis of FOXP3 polymorphisms in infertile women with and without endometriosis. Fertility and Sterility, Birmingham, v. 95, n. 7, p. 2223-2227, 2011.

BACCHETTA, R.; GREGORI, S.; RONCAROLO, M. G. CD4+ regulatory T cells: mechanisms of induction and effector function. Autoimmunity Reviews, Amsterdam, New York, v. 4, p. 491-496, 2005.

BAN, Y.; TOZAKI, T.; TOBE, T.; BAN, Y.; JACOBSON, E. M.; CONCEPCION, E. S.; TOMER, Y. The regulatory $\mathrm{T}$ cell gene FOXP3 and genetic susceptibility to thyroid autoimmunity: an association analysis in Caucasian and Japanese cohorts. Journal of Autoimmunity, London, v. 28, p. 201-207, 2007.

BASSUNY, W. M.; IHARA, K.; SASAKI, Y.; KUROMARU, R.; KOHNO, H.; MATSUURA, N.; HARA, T. A functional polymorphism in the promoter/ enhancer region of the FOXP3/Scurfin gene associated with type 1 diabetes. Immunogenetics, New York, v. 55, n. 3, p. 149-56, 2003.

BJORNVOLD, M.; AMUNDSEN, S. S.; STENE, L. C.; JONER, G.; DAHL-JØRGENSEN, K.; NJØLSTAD, P. R.; EK, J.; ASCHER, H.; GUDJÒNSDÒTTIR, A. H.; LIE, B. A.; SKINNINGSRUD, B.; AKSELSEN, H. E.; RØNNINGEN, K. S.; SOLLID, L. M.; UNDLIEN, D. E. FOXP3 polymorphisms in type 1 diabetes and coeliac disease. Journal of Autoimmunity, London, v. 27, n. 2, p. 140-4, 2006.

BORELLI, S. D.; SOUZA, D.; FREITAS, K. F. F.; BOTINI, F. F.; MELO, F. C.; BEDENDO, J. Comparative study between serological and PCR-SSP methods to determine class II HLA molecules. Acta Scientiarum. Health Science, Brasilia, v. 26, n. 2, p. 337-340, 2004.

BOTTEMA, R. W. B.; KERKHOF, M.; REIJMERINK, N. E.; KOPPELMAN, G. H.; THIJS, C.; STELMA, F. F.; SMIT, H. A.; BRUNEKREEF, B.; VAN SCHAYCK, C. P.; POSTMA, D. S. X-chromosome Forkhead box P3 polymorphisms associate with atopy in girls in three Dutch birth cohorts. Epidemiology and Genetics, Copenhagen, v. 65, p. 865-874, 2010.

CHEN, X.; OPPENHEIM, J. J. Resolving the identity myth: key markers of functional CD4+Foxp3+ regulatory T cells. International Immunopharmacology, Amsterdam, v. 11, n. 10, p. 1489-96, 2011.

COFFER, P. J.; BURGERING, M. T. Forkhead-box transcription factors and their role in the immune system.
Nature Reviews Immunology, London, v.4, p. 889-899, 2004.

DAVIDSON, T. S.; SHEVACH, E. M. Polyclonal Tregs modulate T effector cell trafficking. European Journal of Immunology, Baltimore, v. 41, n. 10, p. 2862-70, 2011.

EASTELL, T.; HINKS; A.; THOMSON, W. SNPs in the FOXP3 gene region show no association with Juvenile Idiopathic Arthritis in a UK Caucasian population. Rheumatology, Basel, v. 46, p. 1263-1265, 2007.

FODOR, E.; GARACZI, E.; POLYÁNKA, H.; KORECK, A.; KEMÉNY, L.; SZÉLL, M. The rs3761548 polymorphism of FOXP3 is a protective genetic factor against allergic rhinitis in the Hungarian female population. Human Immunology, New York, v. 72, n. 10, p. 923-929, 2011.

FONTENOT, J. D.; GAVIN, M. A.; RUDENSKY, A. Y. Foxp3 programs the development and function of CD4+CD25+ regulatory $\mathrm{T}$ cells. Nature Immunology, New York, v. 4, p.330-336, 2003.

GAO, L.; LI, K.; LI, F.; LI, H.; LIU, L.; WANG, L.; ZHANG, Z.; GAO, T.; LIU, Y. Polymorphisms in the FOXP3 gene in Han Chinese psoriasis patients. Journal of Dermatological Science, Amsterdam, v. 57, n. 1, p. 51$56,2010$.

HOFFMAN, P.; BOELD, T. J.; EDER, R.; HUEHN, J.; FLOESS, S.;WIECZOREK, G.;OLEK, S.;DIETMAIER, W.; ANDREESEN, R.; EDINGER, M. Loss of FOXP3 expression in natural human $\mathrm{CD} 4+\mathrm{CD} 25+$ regulatory $\mathrm{T}$ cells upon repetitive in vitro stimulation. European Journal of Immunology, Weinheim, v. 39, p. 1088-1097, 2009.

HORI, S. Developmental plasticity of Foxp3+ regulatory T cells. Current Opinion in Immunology, London, v. 22, p. 575-582, 2010.

HORI, S.; NOMURA, T.; SAKAGUCHI, S. Control of regulatory $\mathrm{T}$ Cell development by the transcription factor Foxp3. Science, New York, v. 299, n. 5609, p. 10571061, 2003.

INOUE, N.; WATANABE, M.; MORITA, M.; TOMIZAWA, R.; AKAMIZU, T.; TATSUMI, K.; HIDAKA, Y.; IWATANI, Y. Association of functional polymorphism related to the transcriptional level of FOXP3 with prognosis of autoimmune thyroid diseases. Clinical and Experimental Immunology, Oxford, v. 162, p. 402-406, 2010.

JONULEIT, H.; SCHMITT, E. The regulatory T cell family: distinct subsets and their interrelations. The Journal of Immunology, Baltimore, v. 171, p. 6323-6327, 2003. 
KHATTRI, R. et al. An essential role for Scurfin in CD4+CD25+ T regulatory cells. Nature Immunology, New York, v. 4, p. 337-342, 2003.

LIN, Y. C.; LEE, J. H.; WU, A. S.; TSAI, C. Y.; YU, H. H.; WANG, L. C.; YANG, Y. H.; CHIANG, B. L. Association of single-nucleotide polymorphisms in FOXP3 gene with systemic lupus erythematosus susceptibility a case-control study. Lupus, Houndmills, v. 20, p. 137-143, 2011.

MELO, K. M.; CARVAlho, B. T. C. Células T regulatórias: mecanismos de ação e função nas doenças humanas. Revista brasileira de alergia e imunopatologia, São Paulo, v. 32, n. 5, p. 184-188, 2009.

NAKANISHI, K.; SHIMA, Y. No contribution of a GT microsatellite polymorphism in the promoter region of the FOXP3 gene to susceptibility to type 1 diabetes in the Japanese population. Clinica Chimica Acta, Amsterdam, v. 384, p. 171-173, 2007.

OWEN, C. J.; EDEN, J. A.; JENNINGS, C. E.; WILSON, V.; CHEETHAM, T. D.; PEARCE, S. H. Genetic association studies of the FOXP3 gene in Grave's disease and autoimmune Addison's disease in the United Kingdom population. Journal of Molecular Endocrinology, Bristol, v. 37, p. 97-104, 2006.

PARK, O. Analysis of the Foxp3/Scurfin gene in Crohn's Disease. New York Academy of Scienses, New York, v. 1051, p. 218-228, 2005.

RASKIN, L.; RENNERT, G.; GRUBER, S. B. FOXP3 germline polymorphisms are not associated with risk of breast cancer. Cancer Genetics and Cytogenetics, New York, v. 193, p. 40-42, 2009.

RUDENSKY, A. Y. Regulatory $\mathrm{T}$ cells and Foxp3. Imunological Reviews, Copenhagen, v. 241, p. 260-268, 2011.

SAKAGUCHI, S.; YAMAGUCHI, T.; NOMURA, T.; ONO, M. Regulatory T cells and immune tolerance. Cell, Cambridge, v. 133, p. 775-787, 2008.

SAKAGUCHI, S. Regulatory T cells: Meden Agan. Immunological reviews, Copenhagen, v. 212, p. 5-7, 2006.

SHEVACH, E. M. Mechanisms of Foxp3+ T regulatory cell-mediated suppression. Immunity, Cambridge, v. 30, p. 636-645, 2009.

TONE, M.; GREENE, M. Cooperative regulatory events and Foxp3 expression. Nature Immunology, New York, v. 12 n. 1, p. 14-16, 2011.

VAN DER VLIET, H. J.; NIEUWENHUIS, E. E. IPEX as a result of mutations in Foxp3. Clinical and Developmental Immunology, 2007.
WHITE, B. N.; DE LUCCA, F. L. Preparation and analysis of RNA. In: TURNER, R. B. (Org.). Analytical Biochemistry of Insects. Amsterdam: Elsevier Scientific Publishing Company, 1977. p. 85-130.

WILDIN, R. S.; SMYK-PEARSON, S.; FILIPOVICH, A. H. Clinical and molecular features of the immunodysregulation, polyendocrinopathy, enteropathy, $\mathrm{X}$ linked (IPEX) syndrome. Journal of Medical Genetics, London, v. 39, n. 8, p. 537-545, 2002.

WU, Z.; YOU, Z.; ZHANG, C.; LI, Z.; SU, X.; ZHANG, $\mathrm{X}$.; LI, Y. Association between functional polymorphisms of Foxp3 gene and the occurrence of unexplained recurrent spontaneous abortion in a Chinese Han population. Clinical and Developmental Immunology, Abingdon, 2012.

ZAVATTARI, P.; DEIDDA, E.; PITZALIS, M.; ZOA, B.; MOI, L.; LAMPIS, R.; CONTU, D.; MOTZO, C.; FRONGIA, P.; ANGIUS, E.; MAIOLI, M.; TODD, J. A.; CUCCA, F. No association between variation of the FOXP3 gene and common type 1 diabetes in the Sardinian population. Diabetes, New York, v. 53, p. 1911-1914, 2004

ZHANG, L.; ZHANG, Y.; DESROSIERS, M.; WANG, C.; ZHAO, Y.; HAN, D. Genetic association study of FOXP3 polymorphisms in allergic rhinitis in a Chinese population. Human Immunology, New York, v. 70, n. 11, p. 930-934, 2009.
Recebido em 27 de março de 2012 Aceito em 23 de julho de 2012 